REVIEW

\title{
Evo-devo of child growth II: human life history and transition between its phases
}

\author{
Ze'ev Hochberg \\ Meyer Children's Hospital, Rambam Medical Center, and Technion-Israel Institute of Technology, PO Box 9602, Haifa 31096, Israel \\ (Correspondence should be addressed to Z Hochberg; Email: z_hochberg@rambam.health.gov.il)
}

\begin{abstract}
This review attempts to use evolutionary life-history theory in understanding child growth in a broad evolutionary perspective. It uses the data and theory of evolutionary predictive adaptive strategies for transition from one life-history phase to the next, and the inherent adaptive plasticity in the timing of such transitions. Humans evolved to withstand energy crises by decreasing their body size, and evolutionary short-term adaptations to energy crises utilize a plasticity that modifies the timing of transition from infancy into childhood, culminating in short stature at the time of an energy crisis. Transition to juvenility is part of a strategy of conversion from a period of total dependence on the family and tribe for provision and security to self-supply, and a degree of adaptive plasticity is provided and determines body composition. Transition to adolescence entails plasticity in adapting to energy resources, other environmental cues, and the social needs of the maturing adolescent to determine lifespan and the period of fecundity and fertility.

Conclusion: Life-history transitions are the times when the child adaptively responds to environmental cues in order to enhance growth-body composition-lifespan-fecundity schedules and behavioral strategies that yield the highest fitness in a given environment.
\end{abstract}

European Journal of Endocrinology 160 135-141

\section{Introduction}

Life-history evolutionary theory is considered here as part of evolutionary development biology (evo-devo) (1) as it addresses factors that produce variation in life histories, which are found both among and within species. Here, it is taken into the realm of clinical medicine and child growth, following the first article in this 'evo-devo' series (2). In the case of humans, life-history evolutionary theory is best understood in the context of biological rationale and cultural expressions as a solution to an ecological problem posed by the environment and subject to constraints intrinsic to the organism (3). Among the questions posed are: why are organisms small or large? Why do they mature early or late? Why do they have few or many offspring? Why do they have a short or a long lifespan? Why must they grow old and die? Several reviews on this topic include $(2,4-7)$.

The transition from one life-history phase to the next requires a mechanism for the onset of the latter. Thus, the transition from infancy to childhood is associated with the setting in of the dominance of the GH-insulin-like growth factor 1 (IGF1) axis. The transition into juvenility requires the development of androgen-generating adrenal reticularis. The beginning of adolescent-related puberty is a function of the hypothalamic-pituitary-gonadal (HPG) axis maturation.
This review presents the data and theory of evolutionary predictive adaptive strategies for transition from one life-history phase to the next and the inherent adaptive plasticity in the timing of such transitions in order to match not only energy supply, but also other environmental cues.

\section{Child growth and the theory of life history}

Life history has been defined as the strategic allocation of an organism's energy toward growth, maintenance, reproduction, raising offspring to independence, and avoiding death $(3,7)$. For a mammal, it is, among others, the strategy of when to be born, when to be weaned, when to stop growing, when to reproduce, and when to die in the best way as to increase its fitness (8).

\section{Life-history phases}

During the evolution of the hominin, childhood and adolescence have been added as new life-history phases (Fig. 1, Table 1) (3). Thus, Homo sapiens have four prolonged and pronounced postnatal pre-adult lifehistory phases: infancy, which lasts for 30-36 months 


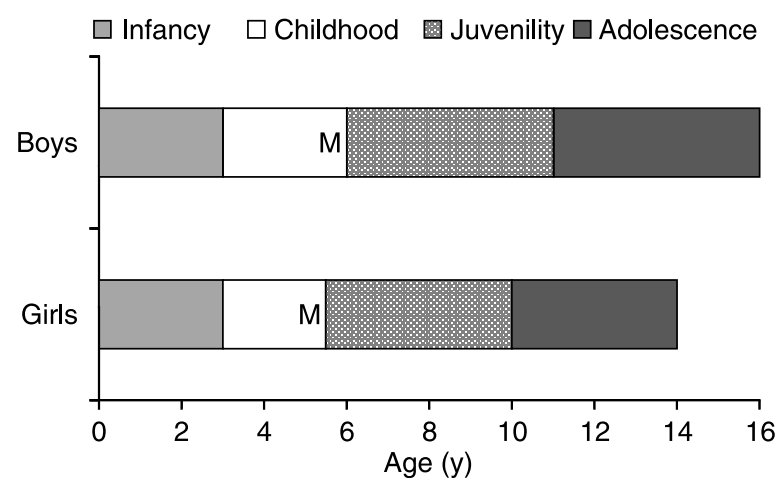

Figure 1 Pre-adult life history for boys and girls. Childhood and adolescence are relatively late development in human evolution. The first molar tooth (M) erupts in other apes at the end of infancy and in humans at the end of childhood.

and ends with weaning from breast feeding in traditional societies; childhood, which lasts for an additional 2-4 years, culminating in a degree of independence for protection and food provision; a juvenile stage of 3-4 years that concludes with the readiness for a sexual maturation process (9); and adolescence, which lasts for 3-5 years, culminating in fertility at an average age of 18 (10).

By reconstruction and without certitude, it has been suggested that some 3000 000-4000 000 years ago the early hominin Australopithecus afarensis had merely two postnatal, pre-adult life-history phases: infancy and juvenility $(7,11)$. He had a long infancy of about 6 years (as compared with 5 years of the chimpanzee), but a comparatively short lifespan, and his first molar tooth erupted in mid-infancy (Fig. 1). With a marked growth of his brain size, 1900000 years ago Homo habilis had to be born less mature to fit into a narrow bipedal maternal pelvis. He had a shorter period of infancy and developed a new strategic life-history phase: childhood, defined by weaning from breast feeding, slowing and stabilization of growth velocity, dependence on older people for food provision and protection, with the first molar erupting at the transition from infancy to childhood. While the brain size steadily grew, and hence newborns had to be born less mature to fit into the narrow birth canal, infancy grew shorter and childhood has been thriving and extending, with the first molar progressively deferred in $H$. sapiens into the transition from childhood to juvenility. Adolescence, as reconstructions suggest, came about as late as 100000 years ago as a distinct life-history phase, with the typical pubertal growth spurt and rapid sexual puberty, deferring the assumption of adulthood in a species that lives longer. The entire assemblage has been extremely successful, and the resulting overpopulation of humans is, for better or for worse, the consequence of that winning strategy.

\section{Transitions between life-history phases}

Staging has retained a central place in evolutionary lifehistory theory and in endocrine deliberation, and rightly so. In defining a life phase for evolutionary, physiological, social, or endocrine inquiries, one inevitably needs to define the stage's beginning and end. Onsets are a key concept of that narrative, whereas offsets remain even more intricately difficult to characterize. Offsets of life-history phases may not really exist.

Multiple hormone mechanisms have evolved to activate both physiological and behavioral traits at the right time and in the correct context as the child transits from infancy to childhood, then to juvenility, and later to adolescence and adulthood. As the child transits from infancy to childhood, the GH-IGF1 sets in and new behavioral traits develop, but the metabolic forces that drive infantile growth remain as active and the infantile mental achievement develop further, although some infantile manners recede while the child ceases to feed on mother's milk. At adrenarche, androgens come into play for the first time, and behaviors change, but the GH-IGF1 axis remains as imperative, while the juvenile joins adults in their daily activities. Adolescence is associated with the onset of the hypothalamic-pituitary-gonadal axis, while both adrenal androgens and the GH-IGF1 axis do not set off secretion.

Endocrine mechanisms are available to set off any of these means in adjusting to environmental signals: malnutrition will slow the GH-IGF1 axis, anorexia

Table 1 Life-history phases, growth milestones, and social function.

\begin{tabular}{ll}
\hline Approximate age & Characteristics of life-history phases \\
\hline 0-30 months & Infancy: breast-feeding \\
& Feeding by maternal lactation. Rapid and decelerating growth. Rapid growth of the brain. Deciduous dentition. \\
& More than $50 \%$ of resting metabolic rate is devoted to brain growth and function \\
& Childhood: dependence for food and protection \\
Slowing and stabilization of the growth rate. Immature dentition. Extended family and tribal care. Adiposity \\
rebound. Dependence on family for food and security. Immature motor control. Cognitive advancement \\
Juvenility: initial independence \\
Adrenarche. Finding much of own food. Avoiding predators. Mid-childhood transition, then slowing growth \\
rate. Competing with adults for food and space. Joining society functions/elementary school \\
Adolescence: preparation for adulthood, non-fertile \\
Dimorphic secondary sexual characteristics. Growth acceleration. Peer-grouping. Stature and muscularity are \\
still pre-adult size. Learn and practice adult skills while still infertile
\end{tabular}


nervosa will block adrenal androgen secretion, and physical exertion will slow the HGP axis, to name just three possibilities.

\section{Phenotypic plasticity and adaptation}

Humans live under a variety of environmental conditions unprecedented in nature, including the entire range of geographical latitudes and altitudes, as well as extremely diverse weather conditions. Whereas the above vary slowly, nutritional conditions may change more rapidly, and evolution has provided for mechanisms to adapt to these extremes, and socio-cultural adjustments filled the remaining gaps when changes were faster than the evolutionary time scale. The secular trend in child growth and puberty is a dazzling example of such an adaptation.

As a consequence of life conditions under changing environment, children may be stunted for short or longer periods, be underweight or overweight, and be at risk for disease. In the endocrine jargon, this has been labeled 'developmental programming.' The evolutionary language for the same is 'predictive adaptive response' (12). A life-history theory analysis rephrases these to the concept of 'trade-offs' (5). Under adverse conditions, Bogin et al. argue, trade-offs result in reduced survival, poor growth, constraints on physical activity, and poor reproductive outcomes (5).

Whereas programming implies permanent maladaptive effects that place people at risk for disease, predictive adaptation considers the phenotypic changes as adaptive, and at two levels: i) short-term adaptive responses for immediate survival and ii) predictive responses required to ensure postnatal survival to reproductive age (fitness). Organismal size, including child growth, is probably the supreme case in point of the paradigm. Franz Boas (1858-1942) introduced the evolutionary concept of developmental plasticity early in the 1900s; this was expanded in the 1960 by Lasker (13), and Gluckman and Hanson suggested the concept of predictive adaptive response $(12,14)$, postulating that it is the mismatch between intrauterine stunting and early infantile catching up that results in the ensuing metabolic morbidity. Predictive adaptive responses require trade-offs, such as reduced fetal growth in favor of survival, or early puberty with lower quality offspring against slow maturation and later first pregnancy with higher quality offspring. A given genotype can generate multiple phenotypes depending on the environmental conditions experienced by the organism during development (15).

\section{Infancy-childhood transition: determination of adult stature}

As recently reviewed, it is the transition from infantile to childhood growth that determines ultimate adult height (2). Based on the predictive adaptive response theory, the expected response to a secure environment includes the investment in large body size, whereas the expected responses to a threatening environment will include a reduction in body size.

For the infancy-childhood growth transition to occur, the child must have a positive energy balance. The infancy-childhood growth transition age is influenced and delayed by disease, when energy consumption increases rapidly (2), and by undernutrition, gastrointestinal infection, and socioeconomic impediments, when energy supplies are scarce $(16,17)$.

There seems to be sufficient indirect evidence to propose that a delay in the infancy-childhood growth transition is a predictive strategy in adapting to an energy crisis during infancy. Yet, the contemporary crisis of Western societies is energy saturation rather than shortage, and the transition from infancy to childhood is also the age of shifting dietary saturation in affluent societies. In children with delayed infancychildhood transition (DICT) (2), mismatching of the predictive adaptive response and otherwise overfeeding results in the unwanted permutation of obesity with short stature (18). This is well documented for migrants from developing to richer countries, who are shorter but at higher risk for overweight and obesity (19). The trade-off approach would explain these intergenerational effects: physical and social abuse lead to pathology rather than adaptation.

Unlike the large volume of experimental data available for intrauterine programming for adult disease (20), the infancy-childhood growth transition cannot be investigated in experimental animals; as mentioned, $H$. sapiens is the only remaining hominin to have a childhood. Further investigation on this topic will have to rely on clinical research.

\section{Childhood to juvenility transition: determination of body composition}

As recently reviewed, it is difficult to decide when to set the transition age to juvenility, as some parts of the juvenile package may start early, and others a year or two later (9). Transition into juvenility may be defined by adrenarche, for the onset of adrenal androgen production at age 5-6 years, by tooth eruption, growth pattern, and the adiposity rebound.

For the paleoanthropologist, the transition from childhood to juvenility is associated with the eruption of permanent molars at age 6 years. A comparative study across 21 primate species found the age of first molar eruption to be highly associated with brain weight $(r=0.98)$ and a host of other life-history variables (21). The study concluded that the mean age of tooth eruption is a good overall measure of the maturation rate of a species, and in the context of the present discussion - the transition from childhood 
to juvenility. If this is the case, then data for dental eruption in 1837 , which showed similar eruption ages to those known today (22), seem to indicate that the onset of juvenility has not changed much over the last 170 years. At a time of a marked worldwide upward trend for height, there has been little change in the timing of tooth eruption (23).

Another possible definition of transition to juvenility derives from the growth curves (Fig. 2). After a period of constant growth rate, the peak of the "mid-childhood spurt' (greater for girls than boys) signifies a transition into a new growth decelerating phase at age 4.5 years for girls and 5.5 years for boys. One possible consequence of this aspect of the transition is that later juvenility onset allows for a longer period of childhood, with its relative stable and high growth rate before the typical juvenile slow down. A year of boys' CJ transition delay contributes to their final height an average of $6 \mathrm{~cm}$. Thus, every month of delay in juvenility onset will advance boys' final height by $0.5 \mathrm{~cm}$ and girls' by close to $0.6 \mathrm{~cm}$.

Adiposity rebound is another indicator for the transition to juveniles' body composition. The adiposity rebound corresponds to the second rise in the agerelated body mass index curve that occurs between ages 4 and 6 years. Its sexual dimorphism corresponds very well to that observed in the second derivative growth curves, with mean boys' rebound at age 68 months, as

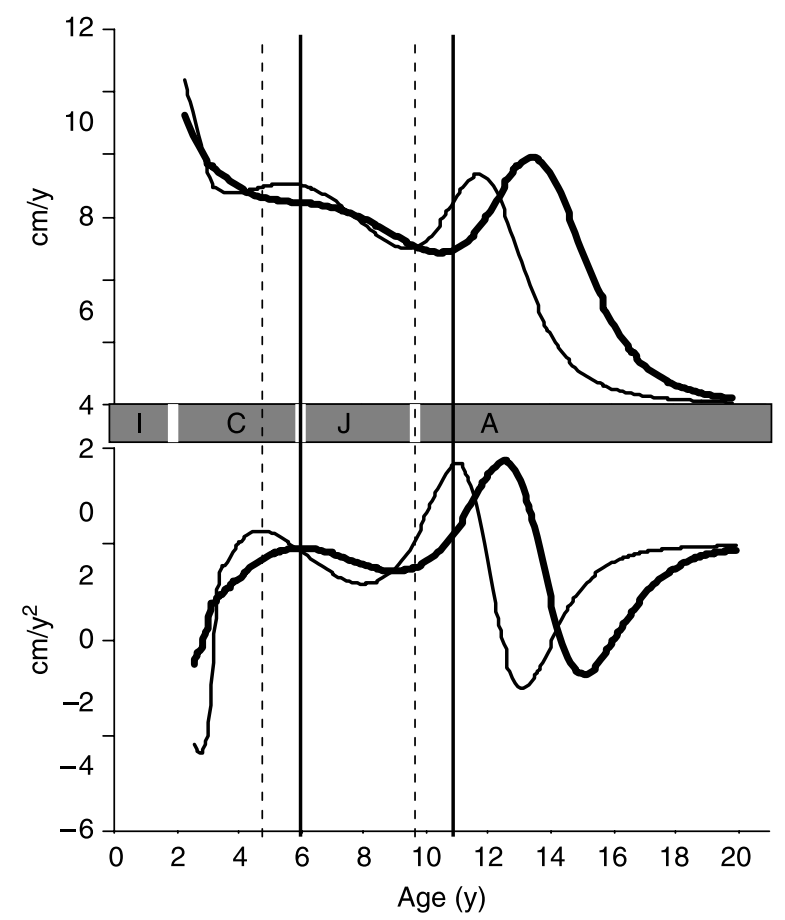

Figure 2 Mean growth velocity (upper panel), and growth acceleration (cm/ $\mathrm{y}^{2}$, lower panel) for boys (solid lines) and girls (soft lines). The first and second derivatives were calculated from (37). I, infancy; C, childhood; J, juvenility and A, adolescence. compared with girls with a mean adiposity rebound 6 months earlier.

Adiposity rebound may be the first clinical sign of juvenility, or it may be the signal that turns the transition on. It is interesting to note that even lean girls with precocious adrenarche have higher levels of IGF1, IGFBP3, and leptin (24) than control, as mechanisms that may transmit the signal of adequate energy readiness.

\section{Transition from juvenility to adolescence}

The onset of puberty has been the topic of much research and many review articles. This will be mentioned here only briefly, with respect to life-history evolutionary perspective and the context of the transition from juvenility to adolescence as a period of adaptive plasticity. Using the paradigm of this review, I speak of a transition from juvenility to adolescence rather that an onset. This semantic matter entails two aspects that are introduced here. Whereas 'onset' implies an event that has happened at once, transition is rather a course of actions that starts during juvenility, progressively gaining power as the child enters a fullscale adolescence. The second non-semantic matter is that the child transits into adolescence, and hypothalamic-pituitary-gonadal maturation with its biological effects is but one aspect of the package known as adolescence. Adaptation of adolescence to the environment and the consequences of early or late transition involve the many facets of adolescence.

Even during childhood and juvenility, girls have higher estrogen levels than boys (25). The onset of puberty in girls is mostly considered to take place when breast buds erupt. However, it is now recognized that this is not the first sign of maturation of the female hypothalamic-pituitary-gonadal axis. Very much like boys, who exhibit their gonads for direct palpation and show testicular growth, the ovaries start to grow discretely about 2 years before breast buds appear. The same is true for estradiol levels, which show a rise about 2 years before thelarche (26), and growth accelerates at least 6 months before breast buds are evident. Boys show a similar pattern of gradual maturation of the hypothalamic-pituitary-gonadal axis. Testosterone levels start to gradually rise at a mean age of 9 , long before gonadal growth - the so called gonadarche (26).

Just as much as the secular trend in human size has been an adaptive phenomenon for an encouraging environment, the receding age of adolescence and pubertal development has been an adaptive response to positive environmental cues in terms of energy balance. The ever-younger age of girls' thelarche and menarche may have more than a single justification. In the last decade, the popular explanation has been that this phenomenon results from environmental exposure to endocrine disruptors, thus accelerating hypothalamic 
maturation. Whereas it may have a bearing on the earlier age of thelarche, which is a recent trend, it can hardly explain the secular trend in the age of menarche over the last 170 years. An evolutionary perspective on this worldwide trend has been proposed by Gluckman and Hanson (27). They have challenged the concept that this has been pathological, and suggested that reproductive and life-history strategies might be reflected in the more frequent presentation of females with early onset adolescence.

With respect to puberty, women face a trade-off between spending a long time accumulating resources through childhood growth, thereby improving the odds for successful pregnancy, against beginning reproduction and increasing the number of reproductive cycles. It turns out that a later first birth allows for a longer period of adolescent weight gain, and that heavier women in traditional societies are more fertile, both correlating with higher birth rates. This trade-off has been used to model the optimal age at first birth. The optimal age at first birth under such models is 18 years, near the observed mean of 17.5 years in such societies (28).

The environmental cues for the transition into adolescence vary with species and gender, and it may be related to altitude, temperature, humidity, and lighting, but mostly it relates to energy balance. Sensors in the hypothalamus and hindbrain monitor these signals and permit high-frequency GNRH1 release when the signals reach appropriate levels (29). The consequences of puberty and adulthood that follow, such as the defense of territory or mate, pregnancy, and care of young, are energetically expensive. The individual must sense whether he/she has grown sufficiently (through metabolic cues), what his/her relationship is to other individuals (through social cues), and whether conditions are optimal to begin the reproductive process (through environmental cues) (30). Metabolic fuel availability, insulin, glucose, and leptin in females serve as important signals for the attainment of somatic growth sufficient to support pregnancy (31).

Consumption of more nutritious foods, such as those derived from animal protein, increased by $\sim 2600000$ years ago when an early hominin displayed an important behavioral shift relative to ancestral forms: the recognition that a carcass represented a new and valuable food source. The shift in the hominin 'prey image' to the carcass and the use of tools for butchery increased the amount of protein and calories available, irrespective of the local landscape. Life-history theory claims that 2600000 years ago was before hominin had an adolescent stage, and a childhood stage had just started to evolve $(7,8)$; the age range of $3-5$ years was that of childhood, and in the absence of adolescence, the transition to juvenility might have determined the age of fertility.

In the past few decades, we have been facing a unique juvenility-adolescence transition where puberty has advanced but psychosocial adolescence has been delayed. Physical growth and the hypothalamicpituitary-gonadal axis start their transit into maturation at the end of the first decade of life, but as late as the second and early third decades of life, adolescents are not mature enough to assume adult roles (27). Biological maturation has come to precede psychosocial maturation significantly for the first time in our evolutionary history, and this 'developmental mismatch,' as Gluckman and Hanson described it, has considerable societal implications. This remains beyond the scope of this review, which focuses on aspects of child growth.

The juvenile-adolescence transitional slowing growth rate has been suggested to be a predictive adaptive period, and the response has to do with longevity in a negative relationship with nutrition during this transition (32); poor food supply results in longer life. Moreover, food supply during this transition had an effect that lasted for at least the children and grandchildren generation, and interestingly enough, it was inherited from fathers to their sons and daughter (32). Using the Northern Sweden Överkalix cohorts of 1890, 1905, and 1920, this study analyzed food supply effects on offspring and grandchild mortality relative risk, using 303 probands and their 1818 parents and grandparents. Paternal grandfather's food supply was linked to the mortality relative risk of grandsons, while

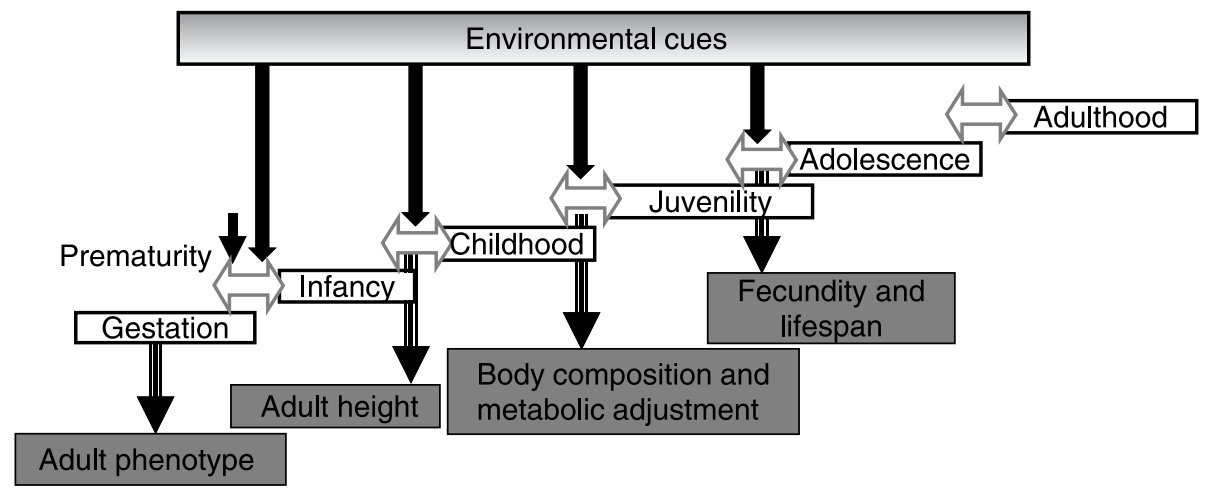

Figure 3 Periods of adaptive plasticity in the transition between life-history phases (double arrows). Transition from infancy to childhood endows with a predictive adaptive response that determines adult height. Transition from childhood to juvenility bestows a predictive adaptive response that resolves adult body composition and metabolic consequences. The transition from juvenility to adolescence establishes longevity and the age of fecundity and reproduction. 
paternal grandmother's food supply was associated with the granddaughters' mortality. These transgenerational effects were observed with exposure during the juvenility-adolescence transition (both grandparents) or fetal/infant life (grandmothers), but not during either grandparent's puberty. This mode of inheritance suggests that the transgenerational transmissions are mediated by the sex chromosomes, $\mathrm{X}$ and $\mathrm{Y}$.

\section{Transgenerational inheritance of phase transition}

Prematurity, an obvious change in transition from one life phase to another, is a familial trait (33). This transition period is not dealt with in this treatise. Likewise, the transition into adolescence is a wellknown familial trait without a genetic background, or, at least, such genes have not yet been discovered. I dare speculate that they never will be. The genes express plasticity/changeability but not the plastic changes. We have some unpublished observations that DICT is also a familial trait, and the same is true for the transition into juvenility (34) and precocious pubarche.

These transgenerational traits are adaptive responses to environment circumstances, which used to last longer than a single generation. In any event, modern lifestyles, with rapid changes in world climate and massive rapid population shifts over long distances might have changed the rules, leading to a mismatch between the environment and the phenotype or between several phenotypic phenomena of the lifephase package (12).

Low birth weight is common in developing countries. However, migrant mothers also give birth to smaller infants despite improved healthcare (35), demonstrating the transgenerational influence of their ancestor environment. In a study in rural Guatemala, pregnant women and their offspring received one of two types of nutritional supplements (36). One was a drink called 'atole,' which was enhanced with protein and calories; the other was a drink called 'fresco,' which was enhanced with calories, but less than the calories in the atole drink. The infants and children of the original study received these nutritional supplements until age 7. In the 1990s, women who were part of the original study as children were asked to be part of a follow-up analysis to investigate the transgenerational influences of the original intervention $(5,36)$. The mothers had been measured repeatedly from their birth until age 3 , as were their children. The report shows that the current generation of infants grew faster than their mothers did. Thirty years after the initial intervention, infants of women who as babies received the proteinenergy drink atole grew faster than infants of mothers who received fresco (calorie-rich only).

\section{Conclusions}

Like other organisms, humans evolved to withstand environmental hardships like energy crises by responding in ways that would maintain some evolutionary fitness, even if sub-maximal. The means to do this is a series of predictive adaptive responses that utilize the sensitive times of transitions from one to its next lifehistory phase, each assigned with its own domain (Fig. 3).

The transition from infancy to childhood is assigned with predictive adaptation to the environment of body size. Short-term adaptations to energy crises utilize a plasticity that postpones the timing of transition to reduce height and advances timing to enhance size.

The transition from childhood to juvenility is part of a strategy in the transition from a period of total dependence on the family and tribe for provision and security into self-supply; it is assigned with a predictive adaptive response of body composition and energy metabolism.

The transition from juvenility to adolescence is assigned with lifespan and the age and length of fecundity. It entails plasticity in adapting to energy resources, other environmental cues, the social needs of adolescence, and their maturation to determine fitness directly.

\section{Declaration of interest}

The author declares no conflict of interest.

\section{Funding}

This research did not receive any specific grant from any funding agency in the public, commercial or not-for-profit sector.

\section{References}

1 Muller GB. Evo-devo: extending the evolutionary synthesis. Nature Reviews. Genetics 20078 943-949.

2 Hochberg Z \& Albertsson-Wikland K. Evo-devo of infantile and childhood growth. Pediatric Research 200864 2-7.

3 Bogin B. Patterns of Human Growth. Cambridge: Cambridge University Press, 1999.

4 Bogin B. Evolutionary perspective on human growth. Annual Review of Anthropology 199928 109-153.

5 Bogin B, Silva MI \& Rios L. Life history trade-offs in human growth: adaptation or pathology? American Journal of Human Biology 2007 19 631-642.

6 Hochberg Z. Juvenility in the context of life history theory. Archives of Disease in Childhood 200893 534-539.

7 Smith BH \& Tompkins RL. Toward a life history of the hominidae. Annual Review of Anthropology 199524 257-279.

8 Smith B. Life history and the evolution of human maturation. Evolutionary Anthropology 19921 134-142.

9 Hochberg Z. Juvenility in the context of life history theory. Archives of Disease in Childhood, 2008.

10 Allal N, Sear R, Prentice AM \& Mace R. An evolutionary model of stature, age at first birth and reproductive success in Gambian women. Proceedings. Biological Sciences 2004271 465-470. 
11 Bogin B. The evolution of human growth. In Human Growth and Development, pp 295-320. Ed. N Cameron, Amsterdam: Academic Press, 2002.

12 Gluckman P \& Hanson MA. The Fetal Matrix. Cambridge: Cambridge University Press, 2005.

13 Lasker GW. Human biological adaptability. The ecological approach in physical anthropology. Science 1969166 1480-1486.

14 Gluckman PD \& Hanson MA. Developmental plasticity and human disease: research directions. Journal of Internal Medicine $2007261461-471$.

15 Crespi EJ \& Denver RJ. Ancient origins of human developmental plasticity. American Journal of Human Biology 200517 44-54.

16 Liu Y, Albertsson-Wikland K \& Karlberg J. Long-term consequences of early linear growth retardation (stunting) in Swedish children. Pediatric Research 200047 475-480.

17 Liu YX, Jalil F \& Karlberg J. Growth stunting in early life in relation to the onset of the childhood component of growth. Journal of Pediatric Endocrinology and Metabolism 199811 247-260.

18 Hermanussen M, Garcia AP, Sunder M, Voigt M, Salazar V \& Tresguerres JA. Obesity, voracity, and short stature: the impact of glutamate on the regulation of appetite. European Journal of Clinical Nutrition $20066025-31$.

19 Varela-Silva MI, Frisancho AR, Bogin B, Chatkoff D, Smith PK, Dickinson F \& Winham D. Behavioral, environmental, metabolic and intergenerational components of early life undernutrition leading to later obesity in developing nations and in minority groups in the USA. Collegium Antropologicum 200731 39-46.

20 Barker DJ. Adult consequences of fetal growth restriction. Clinical Obstetrics and Gynecology $2006 \mathbf{4 9} 270-283$.

21 Smith BH. Patterns of dental development in Homo, Australopithecus, Pan, and Gorilla. American Journal of Physical Anthropology $199494307-325$.

22 Saunders E. Teeth a Test of Age. London: Renshaw, 1837.

23 Helm S. Secular trend in tooth eruption: a comparative study of Danish school children of 1913 and 1965. Archives of Oral Biology $1969141177-1191$

24 Guven A, Cinaz P \& Ayvali E. Are growth factors and leptin involved in the pathogenesis of premature adrenarche in girls? Journal of Pediatric Endocrinology and Metabolism 200518 785-791.

25 Klein KO, Baron J, Colli MJ, McDonnell DP \& Cutler GB Jr. Estrogen levels in childhood determined by an ultrasensitive recombinant cell bioassay. Journal of Clinical Investigation $1994942475-2480$.
26 Ankarberg Lindgren C. Testosterone and 17beta-oestradiol secretion in children and adolescents. Assay development, levels for comparison and clinical applications. Goteborg University, 2005.

27 Gluckman PD \& Hanson MA. Evolution, development and timing of puberty. Trends in Endocrinology and Metabolism 2006 17 7-12.

28 Simondon KB \& Simondon F. Mothers prolong breastfeeding of undernourished children in rural Senegal. International Journal of Epidemiology 199827 490-494.

29 Schneider JE. Energy balance and reproduction. Physiology and Behavior 200481 289-317.

30 Sisk CL \& Foster DL. The neural basis of puberty and adolescence. Nature Neuroscience 20047 1040-1047.

31 Warren MP. Effects of undernutrition on reproductive function in the human. Endocrine Reviews 19834 363-377.

32 Pembrey ME, Bygren LO, Kaati G, Edvinsson S, Northstone K, Sjostrom M \& Golding J. Sex-specific, male-line transgenerational responses in humans. European Journal of Human Genetics 200614 159-166.

33 DeFranco E, Teramo K \& Muglia L. Genetic influences on preterm birth. Seminars in Reproductive Medicine 200725 40-51.

34 Pratt JH, Manatunga AK \& Li W. Familial influences on the adrenal androgen excretion rate during the adrenarche. Metabolism 199443 186-189.

35 Drooger JC, Troe JW, Borsboom GJ, Hofman A, Mackenbach JP, Moll HA, Snijders RJ, Verhulst FC, Witteman JC, Steegers EA \& Joung IM. Ethnic differences in prenatal growth and the association with maternal and fetal characteristics. Ultrasound Obstetrics and Gynecology 200526 115-122.

36 Stein AD, Barnhart HX, Wang M, Hoshen MB, Ologoudou K, Ramakrishnan U, Grajeda R, Ramirez-Zea M \& Martorell R. Comparison of linear growth patterns in the first three years of life across two generations in Guatemala. Pediatrics $2004 \mathbf{1 1 3}$ e270-e275.

37 CDC growth charts: United States. http://www.cdc.gov/growthcharts/, 2000

Received 15 October 2008

Accepted 10 November 2008 